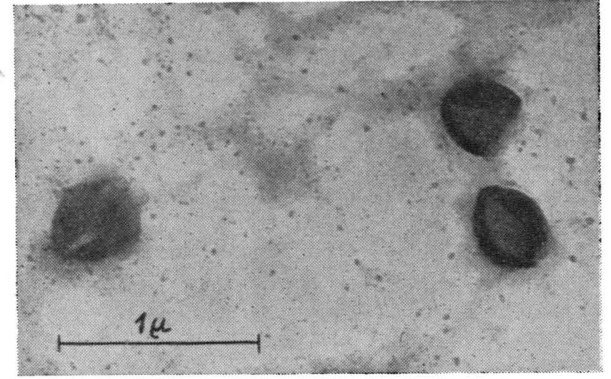

Abb. 2. (5 666/43) Elementarkörper des Virus der Bronchopneumonie der Maus nach H. Ruska (Cystidium Gönnertianum).

Mikroorganismengruppe einzureihen, unabhängig davon, ob das fragliche Virus nur innerhalb geeigneter Wirtszellen züchtbar ist oder nicht. Beim Fleckfiebervirus und anderen Rickettsien ist aus diesen Gründen die Einreihung unter die bakteriellen Mikroorganismen vorgeschlagen worden ${ }^{5,6}$. Eine Einreihung zu einer entsprechenden Organismen-

5 H. Eyer u. H. Ruska, Z. Hyg. Infekt.-Krkh. 125, 483 [1944].

${ }^{6}$ F. Weyer, H. Friedrich-Freksa u. G. Bergold, Naturwiss. 32, 361 [1944]. gruppe muß mit dem Virus der Bronchopneumonie der Maus und wahrscheinlich mit anderen Vertretern der Gruppe der großen Virusarten (Psittakose, Lymphogranuloma inguinale, Trachom) vorgenommen werden.

Die mangelnde Ähnlichkeit der Seiffertschen Mikroorganismen und der ihnen gleichenden Virusformen mit bakteriellen Zellen rechtfertigt die Aufstellung einer neuen Organismengruppe (Klasse oder Ordnung), die wir in Anlehnung an die typischste morphologische Form als Cysticetes (H. Ruska, nov. class.), Cystidiales (nov. ord.) usw. bezeichnen. Sie umfaßt wahrscheinlich die Seiffertschen Mikroorganismen, die Abwasserorganismen von Laid law und Elford, die L-Organismen von Klieneberger, die Erreger der Lungenseuche des Rindes und anderer Lungenerkrankungen der Ratte, der Maus und des Menschen (Her zberg) sowie die oben aufgeführten ,,großen Virusarten“.

Hrn. Prof. Dr. Dr. O. Ẃ aldmann sind wir für die Aufnahme, die das Laboratorium für Übermikroskopie der Siemens \& Halske AG. in der Reichsforschungsanstalt Insel Riems gefunden katte, sehr zu Dank verpflichtet.

\title{
Beiträge zur Genetik des Photoperiodismus
}

\section{Photoperiodismus und Autopolyploidie ${ }^{1}$}

\author{
Von Anton Lang
}

Aus dem Kaiser-Wilhelm-Institut für Biologie, Abt. Melchers, Tübingen (früher Berlin-Dahlem)

(Z. Naturforschg. 2 b, 36-44 [1947]; eingegangen am 14. Februar 1947)

\begin{abstract}
Die Blütenbildung bei autotetraploiden Hyoscyamus niger (Langtagpflanze), Hyoscyamus albus, Antirrhinum majus (Tagneutrale) und Xanthium (Kurztagpflanze) in verschiedenen Tageslängen wurde untersucht. Gegenüber den diploiden Stammformen waren:

bei Hyoscyamus niger die kritische Tageslänge etwas herabgesetzt, Blattzahl und Schoßzeit herab-, die Blütezeit dagegen heraufgesetzt;

bei Hyoscyamus albus das Erscheinen von Knospen und Blüten verzögert, die Blattzahl unverändert;

bei Antirrhinum und Xanthium das Erscheinen von Knospen und (Antirrhinum) Blüten verzögert, die Blattzahl herabgesetzt.

Die Unterschiede waren in allen Fällen quantitativer Art und absolut wie relativ gering.

Die Befunde werden im Hinblick auf einige grundsätzliche Fragen der Polyploidieforschung diskutiert.
\end{abstract}

$\mathrm{E}$ line Veränderung der Entwicklungsgeschwindigkeit und -dauer von Pflanzen im Gefolge experimentell ausgelöster Autopolyploidie ist wiederholt angegeben worden. Es mußte daher auch interessant erscheinen, die photoperiodische Reaktionsweise experimentell hergestellter Autopolyploider zu

1 Am 28. 12. 1944 der ,Zeitschrift für induktive Abstammungs- und Vererbungslehre" eingereicht, aber dort nicht mehr veröffentlicht. untersuchen, um so mehr, als sich photoperiodische Reaktionen aus der Gesamtheit der in der Pflanze ablaufenden Vorgänge verhältnismäßig scharf herauslösen lassen, man also die Wirkung der Pulyploidie auf einen relativ beschränkten und in sich abgeschlossenen Komplex von Vorgängen erfassen kann. In vorliegender Arbeit werden die Ergebnisse von Untersuchungen über die Tageslängenabhängigkeit der Blütenbildung bei Autotetraploiden von 
Hyoscyamus niger (einjährige violettblühende Sippe), Hyosc. albus, Antirrhinum majus (Sippe 50) und Xanthium Strumarium (Sippe X 28) mitgeteilt. Von den diploiden Stammformen der verwendeten Linien verhalten sich innerhalb des natürlichen Tageslängenbereiches unserer Breiten und vnter natürlichen Lichtverhältnissen Hyoscyamus niger als Langtagpflanze, Xanthium als Kurztagpflanze, Hyoscyamus albus und Antirrhinum als Tagneutrale.

\section{Material, Methoden}

Das Hyoscyamus-und Xanthium-Material stammte aus den Kulturen von Dr. G. Melchers und mir; das Material von Antirrhinum wurde mir von Dr. J. Straub und Frau Dr. R. Mattick, geb. Ehrensberger (Kaiser-Wilhelm-Institut für Biologie, Abteilung v. Wettstein), zur Verfügung gestellt. Die Hyoscyamus- und Xanthium-Stämme waren mehrere Generationen lang zur Erhöhung der genetischen Einheitlichkeit in Individualnachzuchten vermehrt worden; die hochgradige genetische Reinheit der Antirrhinum-Sippe 50 ist bekannt. Die tetraploiden Formen waren mittels der Colchicin-Methode hergestellt worden, die der Hyoscyamus-Arten von Dr. B. Györffy ${ }^{2}$, die von Antirrhinum und Xanthium von Dr. Stra ub. Für die Versuche wurden die erste oder zweite Nachzuchtgeneration, also die Kinder oder Enkel der polyploidisierten Pflanzen, verwendet.

Die Versuche wurden in den bei Lang u. Melcher ${ }^{\mathbf{3}}$ beschriebenen Lichtthermostaten unter im Durchschnitt weitestgehend konstanten Licht- und Temperaturbedingungen, bei konstanter Lichtintensität (also mit der Tageslänge abnehmender Lichtmenge) und bei einer Temperatur von $22^{\circ}$ bis $23^{\circ}$ ausgeführt. Infolge des beschränkten Raumes mußte die Zahl der Versuchspflanzen niedrig gehalten werden (4 bis 5 je Gruppe). Da aber die individuelle Variabilität in den meisten Fällen klein war, ist die Genauigkeit der Ergebnisse für das Ziel der vorliegenden Versuche im allgemeinen ausreichend. Bestimmt wurde außer dem Zeitpunkt des Eintretens der Blütenbildung (Beginn der Achsenstreckung, Zeitpunkt des Erscheinens sichtbarer Knospen oder der 1. offenen Blüte) stets auch die Zahl der bis zur Anlage der 1. Blüte gebildeten Blätter. Überall, wo es nicht zur Ausbildung sichtbarer Blüten kam, wurden die Pflanzen bei Ver-

2 Naturwiss. 26, 547 [1938].

3 Planta 33, 653 [1943]. suchsende auf etwaiges Vorhandensein von Blütenprimordien mikroskopisch kontrolliert. In die Versuche kamen die Pflanzen meist in jungem Zustand, kurz nach der Pikiervng, vorher und bei etwaiger längerer Vorkultur befanden sie sich in der niedrigsten Tageslänge des geplanten Versuchs. - An Düchführung und Auswertung der Versuche waren Frl. G. Gelderblom und Frl. H. Kunisch, beide Berlin, beteiligt.

\section{Versuchsergebnisse}

a) Hyoscyamus niger. Hyoscyamus niger wurde in Tageslängen von $8 \mathrm{~h}, 9 \mathrm{~h}, 10 \mathrm{~h}, 11 \mathrm{~h}, 13 \mathrm{~h}, 15 \mathrm{~h}, 18 \mathrm{~h}$ und 24h (Dauerlicht) kultiviert. Im 8h-Tag war cie Entwicklung der Pflanzen deutlich geschwächt, in den übrigen Tageslängen normal. Blütenbildung trat im 11h-Tag und darüber ein (vgl. Abb. 1). Im 11h-Tag wurden dabei in der Versuchszeit (95d) nur mikroskopische Blütenanlagen gebildet; erst im 13h-Tag kamen alle Pflanzen zum Schossen und Blühen. Bej Heraufsetzung der Lichtzeit avf $15 \mathrm{~h}$ ist die zusätzliche Beschleunigung der Schoßzeit und Herabsetzung der Blattzahl viel geringer; weitere Heraufsetzung der Tageslänge hat noch weniger Wirkung. Die photoperiodische Reaktionskurve hat also denselben charakteristischen Verlauf wie die früher ermittelte einer gelbblühenden Rasse der Art (Lang u. Melchers $\left.{ }^{\mathbf{3}}\right)$; die kritische Tageslänge, die etwa zwischen 10 und $11 \mathrm{~h}$ Licht täglich liegt, entspricht offenbar ebenfalls derjenigen dieser Rasse. Das Erscheinen von sichtbaren Knospen und der Beginn der Blüte werden bei Heraufsetzung der Tageslänge relativ stärker beschleunigt als die Schoßzeit; der Abfall ihrer Kurve ist dementsprechend verhältnismäßig weniger steil (Blütezeit vgl. Abb. 1).

Für das Verhalten der Di- und Tetraploiden ergab sich folgendes: In der Blattzahl blieben diese regelmäßig hinter jenen zurück; im Schossen waren sie ihnen etwas voraus; offene Blüten erschienen aber etwas später. Das letzte gilt allerdings nur für höhere Tageslängen (15h Lichtzeit und mehr). Im 13h-Tag begann bei den Tetraploiden auch die Blüte vor den Diploiden; gleichzeitig wurden die Differenzen in Blattzahl und Schoßzeit absolut wie relativ größer (zum Schossen kamen im 11h-Tag bis Abbruch des Versuches nur die Tetraploiden). Das bedeutet, daß bei den Tetraploiden die kritische Tageslänge etwas tiefer liegt als bei den Diploiden, wodurch sich die Entwicklungsverzögerung mit Herabsetzung der Tageslänge bei ihnen etwas später auswirkt. 

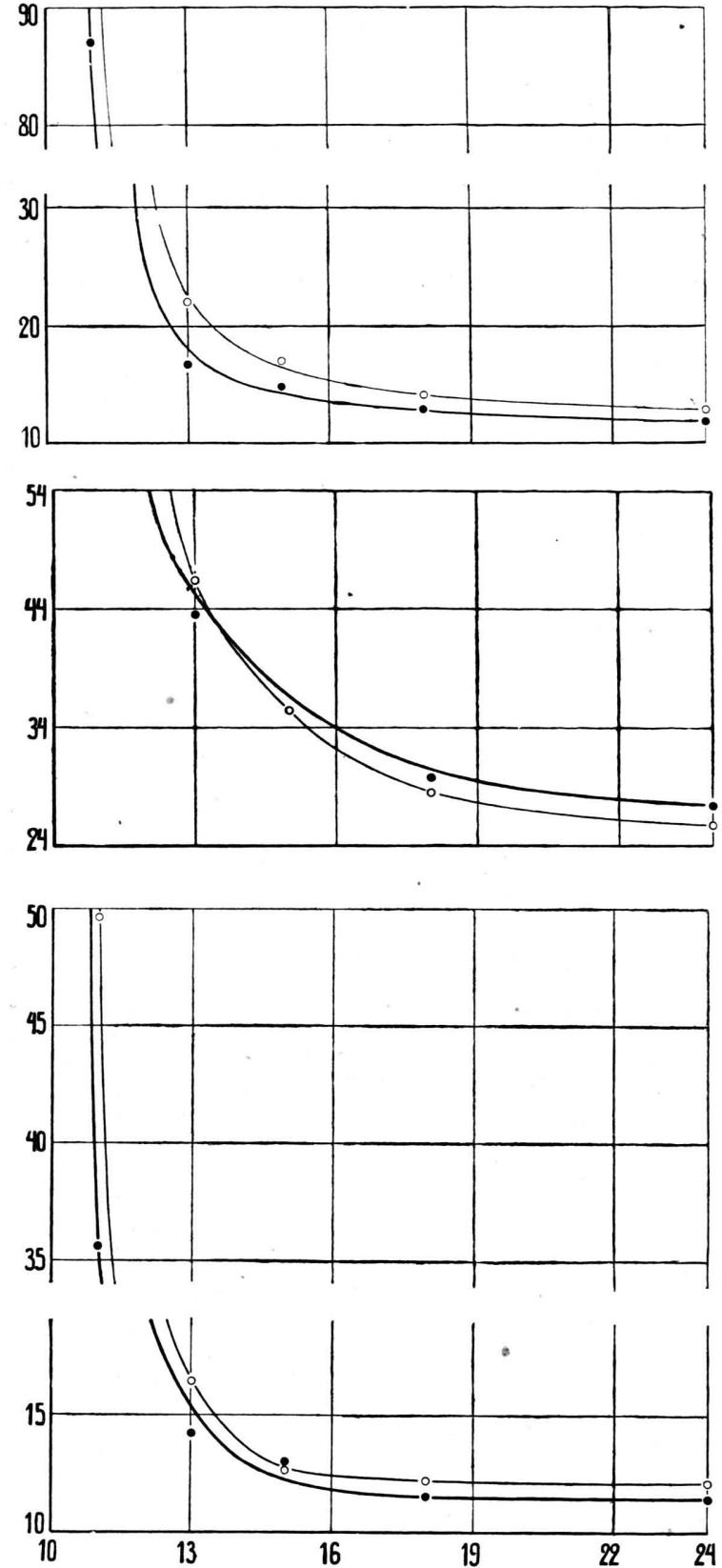

Abb. 1. Hyoscyamus niger, photoperiodische Reaktion von Di- und Autotetraploiden. Oben Schoßzeit, Mitte Blütezeit, I) unten Blattzahl.

Hier und in allen folgenden Abbildungen bedeuten: schwächere Kurven und offene Zeichen: Diploide, stärkere Kurven und ausgefüllte Zeichen: Tetraploide; Abszisse: tägliche Lichtzeit (Stunden); Ordinaten: Tage oder Blattzahlen.

In einemweiteren Versuch, ausgeführt mit älteren, in 9h-Tag vorkultivierten Pflanzen, wurden die Tageslängen enger gestaffelt - unter anderem erhielt eine Versuchsgruppe $10 \frac{1}{2}$ h Licht täglich —, um möglicherweise den Unterschied im Verhalten der Di- und Tetraploiden schärfer zu erfassen. Dies wurde aber nicht erreicht; die Differenzen (festgestellt wurden nur Schoßzeit und Blattzuwachs ${ }^{4}$ ) waren geringer als im zuerst beschriebenen Versuch, so daß sich in den höheren Tageslängen schon von $11 \mathrm{~h}$ an überhaupt keine Verschiedenheit im Verhalten der beiden Polyploidiestufen feststellen ließ. Nur bei $10 \frac{1}{2}$ h Tageslänge war die Entwicklung der Diploiden gegenüber der der Tetraploiden etwas verzögert, was mit dem Ergebnis des vorigen Versuches grundsätzlich in Einklang steht ${ }^{5}$.
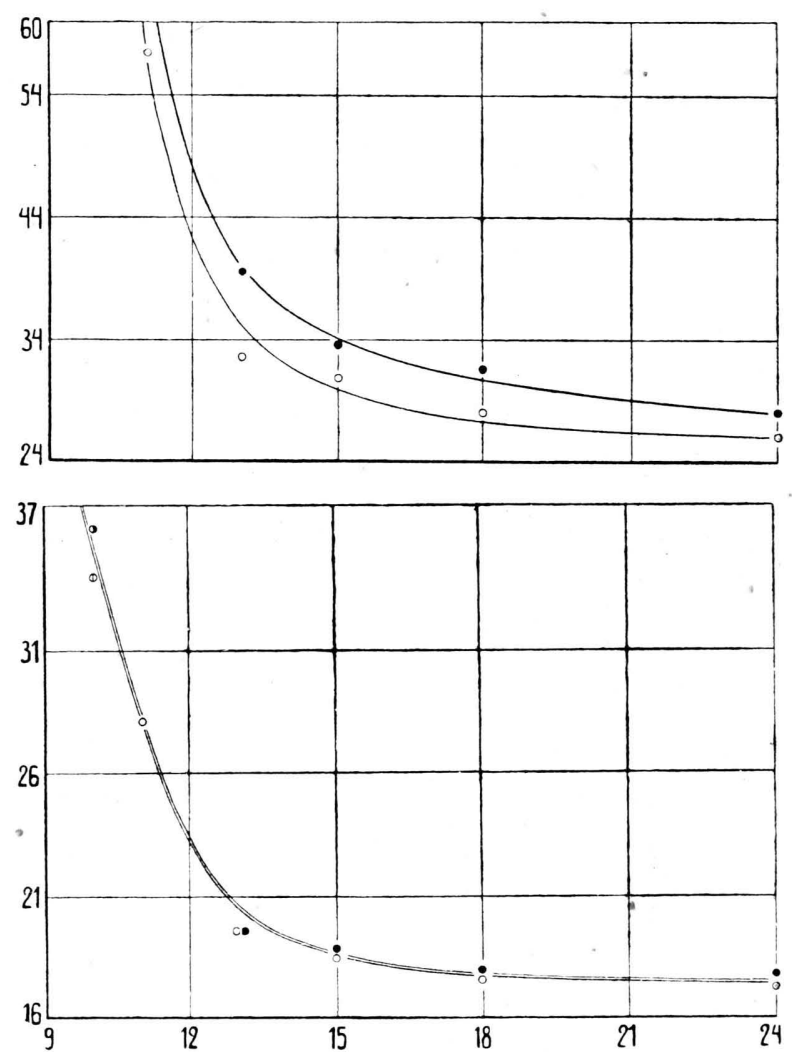

Abb. 2. Hyoscyamus albus, Entwicklung von Di- und Autotetraploiden in verschiedenen Tageslängen. Oben Zeit bis zum Erscheinen von sichtbaren Knospen, unten Blatt. zahlen. Halbierte Zeichen: Blütenbildung nur bei einem Teil der Pflanzen.

${ }^{4}$ Der Blattzuwachs ist die Zahl der vom Versuchsbeginn an gebildeten Blätter; vgl. Lang u. Melchers ${ }^{3}$.

${ }_{5}$ Der verschiedene Ausfall der Versuche ist als solcher nicht überraschend: Das photoperiodische Verhalten der Hyoscyamus-Pflanzen kann infolge des Einflusses innerer Faktoren von Versuch zu Versuch etwas schwanken, und da andererseits die Unterschiede zwischen Di- und Tetraploiden, auch nach dem 1 . Versuch beurteilt, nur gering sind, so können unter bestimmten Umständen diese Unterschiede von anderen Einflüssen überlagert werden und nicht zum Ausdruck kommen. 
b) Hyoscyamus albus. Das Verhalten von Hyoscyamus albus wurde in Tageslängen zwischen 7 und $24 \mathrm{~h}$ beobachtet (vgl. Abb. 2). Im Erscheinen der Knospen und Blüten blieben die Tetraploiden hinter den Diploiden etwas zurück. Die Unterschiede nehmen von hoher zu niederer Tageslänge zu; sie sind nicht groß, die Richtung ist aber gleichmäßig ausgeprägt. In den Blattzahlen waren dagegen keine Unterschiede vorhanden (Abb. 2 unten).

c) Antirrhinum majus. Die Beobachtungen am Löwenmaul (Sippe 50) erstreckten sich auf den Tageslängenbereich von 8-18h (vgl. Abb. 3). Knospen und Blüten erschienen, yie bei Hyoscyamus albus, bei den Tetraploiden etwas verspătet, wobei die Unterschiede - soweit das Material eine Aussage zuläßt - mit abnehmender Lichtzeit ebenfalls größer zu werden scheinen; die Blattzahl war niedriger als die der Diploiden.

Es ist auffällig, daß sowohl bei Antirrhinum wie bei Hyoscyamus albus die Blütenbildung mit zunehmender täglicher Lichtzeit merklich gefördert war und daß sie unterhalb eines gewissen Tageslängenwertes - bei Antirrhinum unter 10h, bei Hyoscyamus albus unter $10-11 \mathrm{~h}$ — völlig ausblieb. Unter den Bedingungen dieser Versuche verhielten sich beide Formen also ähnlich wie Langtagpflanzen. Im S smmer bilden aber im natürlichen Tageslicht

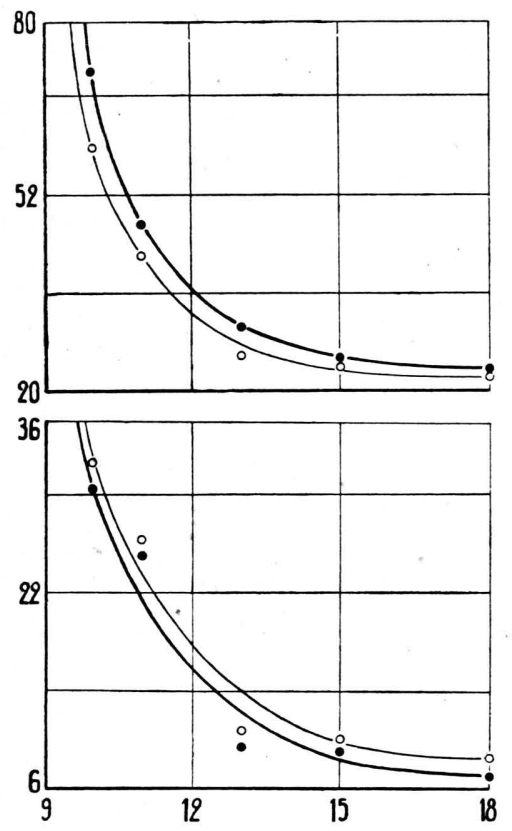

Abb. 3. Antirrhinum majus Sippe 50, Entwicklung von Di- und Autotetraploiden in verschiedenen Tageslängen. Oben Zeit bis zum Erscheinen von sichtbaren Knospen, unten Blattzahlen.
Antirrhinum wie Hyoscyamus albus im 9-10h-Tag mühelos Blüten, Antirrhinum läßt sich auch in unserem Mittwintertag durch Heraufsetzung der Lichtintensität bei unveränderter Belichtungsdauer zur Blüte bringen (vgl. S. 41), während Hyoscyamus niger in solchen Tageslängen - Temperaturen nicht unter $20^{\circ}$ vorausgesetzt - stets vegetativ bleibt. Es hat somit den Anschein, daß bei den sogenannten Tagneutralen die Lichtquantität (und evt1. -qualität) einen relativ viel größeren Einfluß auf die Blütenbildung hat als bei typischen Langtagpflanzen, und daß - im Gegensatz zu diesen - ihre Erhöhung (bzw. Änderung) eine Herabsetzung der Lichtdauer weitgehend kompensieren kann. Bei Tagneutralen würden sich also photoperiodische und photoquantitative Wirkungen zum mindesten überdecken, wenn nicht photoquantitative allein entscheidend sind. Der Verlauf der photoperiodischen Reaktionskurven spricht für eine solche Möglichkeit. Die Kurven fallen weniger steil $a b$ und werden weniger rasch flach als bei Hyoscyamus niger. Die photoperiodischen Reaktionskurven von Hyoscyamus niger und anderen gleichartig reagierenden Langtagpflanzen haben Lang u. Melchers ${ }^{3}$ als Resultante von zwei gegenläufigen Vorgängen gedeutet: Von unmittelbar für die Auslösung der Blütenbildung maßgebenden Primärvorgängen, die, um wirksam zu werden, einen bestimmten Schwellenwert erreichen müssen, und von sekundären Hemmungsvorgängen, die den Primärvorgängen entgegenarbeiten und unterhalb der kritischen Tageslänge deren Wirkung aufheben. Die Primärvorgänge sind versuchsweise mit den Vorgängen der Blühhormonproduktion identifiziert worden, wobei das notwendige Ausgangsmaterial von der Photosynthese geliefert wird, die Sekundärvorgänge mit Dissimilationsprozessen, die in Kurztagsbedingungen auf dieses Ausgangsmaterial übergreifen. Nimmt man an, daß bei einem Objekt die Primärvorgänge, also letztlich die Photosynthese, allein oder doch in wesentlichem Ausmaße für das Eintreten der Blütenbildung entscheidend sind, so würden die resultierenden Kurven - die also nur die Summation einer einzelnen Wirkung ausdrücken würden - den bei Hyoscyamus albus rnd Antirrhinum gefundenen grundsätzlich entsprechen. Allerdings muß angenommen werden, daß auch bei den Tagneutralen, und dann ebenso, auch abgesehen von der Wirkung der sekundären Hemmungsvorgänge, bai Langtagpflanzen, Assimilate für die Blütenbildung erst von einer bestimmten photosyntheti- 
schen Leistung an zur Verfügung stehen, da das Wachstum der Pflanzen bei Herabsetzung der Lichtzeit unter die für Blütenbildung erforderliche Mindestdauer zunächst nicht beeinträchtigt war, ein Lichtmangel also noch keineswegs vorlag. - Während Hyoscyamus niger, solange vegetativ, im $R_{0}-$ settenzustand verharrt, streckten Hyoscyamus albus und Antirrhinum die Achse unabhängig von der Blütenbildung, Hyoscyamus albus gegenüber blühenden Exemplaren vermindert, Antirrhinum sogar in dem gleichen Ausmaß. Auch hierin könnte ein durchgehender Unterschied zwischen Reaktionstypen wie Hyoscyamus niger einer-, Hyoscyamus albus und Antirrhinum andererseits liegen.

d) Xanthium. Xanthium (Sippe X 28) wurde in Tageslängen von $13-16 \mathrm{~h}$ mit $\frac{1}{2}$-stdg. Intervallen, ferner bei $18 \mathrm{~h}, 20 \mathrm{~h}$ und in Dauerlicht kultiviert. Blüten wurden in allen Tageslängen angelegt, in den höheren jedoch wesentlich - zeitlich wie an der Blattzahl gemessen etwa auf das Doppelte - verzögert. Die Verzögerung beginnt bei Lichtzeiten von $14-14 \frac{1}{2} \mathrm{~h}$; zwischen 13 und $14 \mathrm{~h}$ und ebenso über 18h hinaus sind Änderungen der Tageslänge ohne weiteren Effekt. (In den höheren Tageslängen bildeten die Pflanzen nur mikroskopische Infloreszenz- und Blütenprimordien aus, einige blieben sogar ganz vegetativ; das liegt a ber nur an der etwas knapp bemessen gewesenenVersuchsdauer.)Bemerkenswert ist, daß Xanthium X 28 bei Aufzucht in den natürlichen Licht- und Tageslängenbedingungen im Sommer viellänger vegetativ bleibt als in den Thermostaten selbst in Dauerlicht; Blütenbildung tritt dort gewöhnlich erst gegen Herbst, mit stärker abnehmenden Tagen, ein. Auch bei diesem Objekt hat augenscheinlich die Lichtintensität für die Blütenbildung eine wesentliche Bedeutung, und zwar scheinen bei schwächeren Intensitäten lange Belichtungszeiten die Blütenbildung weniger stark zu verzögern als

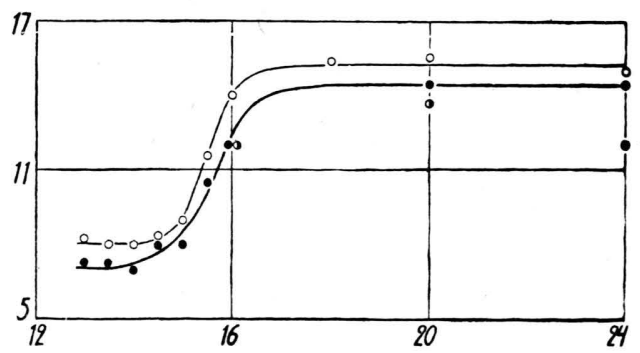

Abb. 4. Xanthium Siṕpe X 28, Blattzahl bei Di- und Autotetraploiden in verschiedenen Tageslängen.

Halbierte Zeichen: Blattzahlen der bis Versuchsende noch nicht zur Infloreszenzanlage gekommenen Pflanzen. bei hohen. Allerdings ist diese Kompensationsmöglichkeit, soweit unsere Beobachtungen schon ein Urteil zulassen, beschränkt; der Kurztagcharakter der Sippe blieb auch bei der künstlichen Beleuchtung deutlich erkennbar.

Das Verhältnis der Tetraploiden gegenüber den Diploiden war dasselbe wie bei Antirrhinum: zeitlich eine leichte Verzögerung, in der Blattzahl eine, gleichfalls geringfügige, Herabsetzung; eine Verschiebung in der Wirksamkeit der Tageslängen war nicht festzustellen (vgl. Abb. 4).

\section{Diskussion \\ I.}

Überblickt man die Ergebnisse der Versuche zusammenfassend, so kann man als Hauptbefund eines feststellen: Die Veränderung der Tageslängenabhängigkeit der Blütenbildung bei experimentell induzierter Autopolyploidie ist geringfügig; die Unterschiede gegenüber den Diploiden sind quantitativ und halten sich absolut wie relativ in engen Grenzen. Meist handelt es sich um eine Verschiebung der Kurven in Richtung der Ordinate: die Vertreter der beiden Polyploidiestufen unterscheiden sich in allen Tageslängen gleichsinnig, wobei die Unterschiede im Bereich des steileren Verlaufs der Kurve größer werden. Bei Hyoscyamus niger ist auch eine Verschiebung in Richtung der Abszisse gegeben, indem die kritischen Tageslängen der Di- und Tetraploiden etwas verschieden sind, mit Annäherung an diesen Bereich sich daher die gegenseitige Beziehung der beiden Typen ändert. Da dies Ergebnis für alle vier untersuchten Objekte und damit für die verschiedenen photoperiodischen Reaktionstypen gilt, darf angenommen werden, daß es den Einfluß $ß$ von Polyploidisierung auf die reproduktive Entwicklung von Blütenpflanzen grundsätzlich repräsentiert.

In der Literatur liegen allerdings zwei Arbeiten, zugleich - soweit sich derzeit übersehen läßt bisher die einzigen Beiträge zu dieser Frage, über das photoperiodische Verhalten von experimentell hergestellten Autopolyploiden vor, die dieser Schlußfolgerung widersprechen, da in beiden Fällen eine stärkere, qualitative Änderung der photoperiodischen Reaktionsweise angegeben wird. Es handelt sich um Beobachtungen an Pflanzen, die im Spätsommer oder Herbst ausgesät wurden und deren Entwicklung (Gewächshauskultur) in die Wintermonate fiel. Ernst ${ }^{6}$ beobachtete unter diesen Bedingungen bei tetraploidem Antirrhinum majus,

${ }^{6}$ Ber. dtsch. bot. Ges. 59, 351 [1941]. 
Sippe 50, eine Hemmung erst der Haupt-, später, nach zunächst kräftiger Entwicklung, auch der Seitentriebe und Ausbleiben der Ausbildung von Infloreszenzen, $0^{6} \mathrm{Mara}^{7}$ bei tetraploiden Pflanzen einer Sommerroggenrasse weibliche oder zygotische Sterilität. Da die zugehörigen Diploiden diese Erscheinungen nicht zeigten und da dieselben sich bei Verlängerung der Lichtzeit mittels Zusatzbeleuchtung aufheben oder vermeiden ließen, wird in beiden Fällen geschlossen, daß mit der Erreichung der $4 n$-Stufe ein neuartiges photoperiodisches Verhalten eingetreten sei.

In beiden Fällen ist freilich diese Schlußfolgerung durch das Beobachtungsmaterial noch in keiner Hinsicht gerechtfertigt. Es kann sich ebensogut um photoquantitative Wirkungen, und zwar um Folgen einfachen Lichtmangels handeln; diese Deutung ist von vornherein sogar die wahrscheinlichere, da Wachstumshemmungen wie die von Ernst gefundenen für das Verhalten von Langtagpflanzen in Kurztagbedingungen durchaus nicht charakteristisch sind, und da es Hinweise für eine Störung der Gametogenese bei Getreiden unter ungünstigen Lichtverhältnissen gibt ${ }^{8}$. Die Beobachtungen von Ernst habe ich am gleichen Material, Antirrhinum majus Sippe 50 2n und 4n, nachgeprüft und habe dabei diesen Zweifel vollauf bestätigt gefunden, darüber hinaus festgestellt, daß zwischen Tetraund Diploiden auch in dieser Eigenschaft nur quantitative, geringfügige Unterschiede bestehen, und außerdem, daß die Wachstumshemmung mit der Blütenbildung nicht zusammenhängt. Im einzelnen wurde folgendes beobachtet (Aussaat der Pflanzen 27. Juli und 1. September, Kultur im Gewächshaus bis 30. Januar):

1. Die von Ernst beschriebene Entwicklungshemmung trat auch bei meinem Material auf, jedoch bei den Diploiden ébenso wie bei den Tetraploiden, nur etwas spätei ${ }^{9}$.

7 American Naturalist 76, 386 [1942].

8 S J. Kraëvoj u. F. G. Kiričenko Doklady Ak. Nauk SSSR 1935 I, 171; G. S.Žu k ova, ebd.19, 297 [1938].

9 Daß bei Ernst die Unterschiede anscheinend viel größer waren, die Diploiden sogar überhaupt keine Hemmung zeigten, ist nicht weiter erstaunlich : Zweifellos können Bedingungen auftreten, oder lassen sich finden, in denen zwischen Di- und Tetraploiden scheinbar qualitative Unterschiede vorhanden sind. $Z$. B. ist es denkbar, da $B$ in meinem ersten Versuch mit Hyoscyamus niger (siehe S. 37) bei einer Tageslänge von etwa $10 \frac{1}{2}$ oder $10 \frac{3}{4}$ Stunden wohl noch die Tetra-, nicht mehr aber die Diploiden zur Blütenanlage gekommen wären; bei Vergleich des Verhaltens allein in dieser Tageslänge hätte sich also auch bei diesem Objekt der Eindruck einer neuartigen photoperiodischen Reaktionsweise der Tetraploiden ergeben.
2. Die Hemmung trat zuweilen vor, zuweilen aber auch erst nach Anlage der Infloreszenz auf; sie beruht offenbar auf einer Sistierung des Wachstums, die ebensogut vor wie nach Beginn der reproduktiven Entwicklung einsetzen kann.

3. Durch zusätzliche Beleuchtung (5 200-WattOsram-Nitra-Lampen über $1 \times 1 \mathrm{~m}$-Glasküvette mit $\sim 5 \mathrm{~cm}$ Wasser) während der Tageslichtstunden, also ohne jede Verlängerung der Lichtzeit, konnte die Hemmung bei Di- wie Tetraploiden vermieden oder wieder aufgehoben werden; sie hat mit der Tageslänge also nichts zu tun. -

Um die Richtung des Einflusses der Autopolyploidie auf die Entwicklung genauer zu bestimmen, muß man sich vergegenwärtigen, daß der tatsächliche Entwicklungsablauf das Produkt von zwei Vorgängen ist, dem allgemeinen Wachstum der Pflanzen, also der Neubildung von Körpersubstanz, und den eigentlichen, spezifischen Entwicklungsvorgängen, und muß versuchen, den Anteil dieser beiden Vorgänge an der beobachteten Entwicklungsänderung zu erfassen. Als Maßstab für die Geschwindigkeit der eigentlichen Entwicklung läßt sich im Falle der reproduktiven Entwicklung die Blattzahl verwenden, da sie die Dauer der vegetativen Phase anzeigt und diese durch das frühere oder spätere Einsetzen der reproduktiven bestimmt wird. Der zeitliche Abla uf der Entwicklung wird dagegen zweifellos weitgehend von der Geschwindigkeit des allgemeinen Wachstums bestimmt. Um diese allgemeine Wachstumsgeschwindigkeit abzuschätzen, wurde bei Hyoscyamus niger, Hyoscyamus albus und Antirrhinum die Geschwindigkeit der Ausbildung von Blättern bestimmt (Auszüge siehe Tab. $1 \leftarrow 3$ ). Bei Hyoscyamus niger blieben die Tetraploiden in der Blattzahl hinter den Diploiden (mit einer Ausnahme) zurück, ihre Wachstumsgeschwindigkeit ist also offenbar verlangsamt. Da die Endblattzahl bei ihnen niedriger war und das Schossen früher eintrat als bei den Diploiden, muß andererseits die eigentliche Entwicklung bei Autotetraploidie beschleunigt sein, und zwar in einem Ausmaß, daß trotz des langsameren Wachstums die reproduktive Entwicklung auch zeitlich eher einsetzt als bei den Diploiden. Nach erfolgtem Übergang in die reproduktive Phase führt das langsamere Wachstum allerdings offenbar wieder zu einem zeitlichen Zurückbleiben der Polyploiden, was sich in ihrer späteren Blütezeit äußert. Bei Hyoscyamus albus waren in der Geschwindigkeit der Blattbildung zwischen den Tetra- und Diploiden keine Unter- 
Tabelle 1

Hyoscyamus niger, Blattzablzunahme bei Di- und Autotetraploiden

\begin{tabular}{|c|c|c|c|c|c|c|c|c|c|c|c|c|c|c|c|c|}
\hline 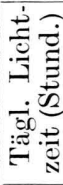 & $\begin{array}{l}\stackrel{0}{0} \\
\frac{0}{0} \\
\frac{0}{2} \\
\frac{\pi}{0} \\
0\end{array}$ & 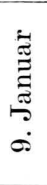 & 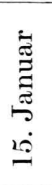 & & 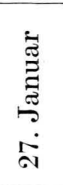 & 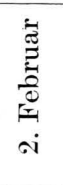 & 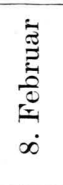 & 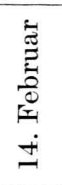 & 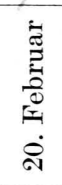 & 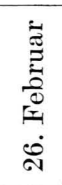 & 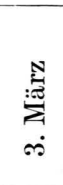 & 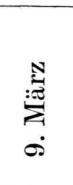 & 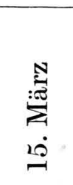 & $\frac{N}{\dot{N}}$ & 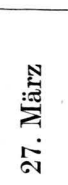 & $\begin{array}{l}\overrightarrow{0} \\
\text { 竞 } \\
10\end{array}$ \\
\hline \multirow{2}{*}{10} & $2 \mathrm{n}$ & 2,0 & 3,4 & 5,4 & 8,0 & 10,2 & 11,8 & 13,6 & 15,4 & 16,4 & 18,8 & 20,6 & 22,4 & 24,4 & 26,2 & 28,8 \\
\hline & $4 n$ & 2,0 & 2,3 & 4,0 & 5,7 & 8,0 & 9,3 & 10,7 & 12,3 & 13,7 & 15,0 & 17,0 & 18,7 & 20,3 & 22,7 & 24,3 \\
\hline \multirow{2}{*}{11} & $2 n$ & 1,6 & 2,3 & 4,3 & 6,3 & 9,3 & 10,3 & 12,7 & 13,7 & 15,7 & 19,0 & 22,3 & 26,0 & 28,0 & 30,3 & 35,0 \\
\hline & $4 n$ & 1,4 & 2,4 & 4,2 & 5,6 & 7,2 & 8,6 & 10,4 & 12,0 & 13,0 & 15,8 & 17,8 & 20,4 & 22,0 & 23,8 & 26,4 \\
\hline \multirow{2}{*}{15} & $2 n$ & 1,8 & 4,0 & 6,2 & 10,0 & 11,6 & $.12,6$ & 12,8 & - & - & - & - & - & - & - & - \\
\hline & $4 n$ & 2,0 & 4,2 & 6,2 & 10,0 & 12,4 & 13,0 & 13,0 & - & - & - & - & - & - & - & - \\
\hline \multirow{2}{*}{18} & $2 n$ & 2,4 & 5,0 & 8,2 & 12,2 & 12,2 & - & - & - & - & - & - & - & - & - & - \\
\hline & $4 n$ & 2,0 & 4,2 & 7,2 & 11,0 & 11,6 & - & - & - & - & - & - & - & - & - & - \\
\hline
\end{tabular}

Tabelle 2

Hyoscyamus albus, Blattzahlzunahme bei Di- und Autotetraploiden

\begin{tabular}{|c|c|c|c|c|c|c|c|c|c|c|c|c|c|c|c|}
\hline 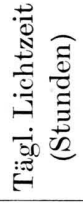 & 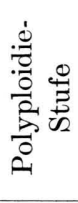 & 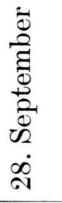 & 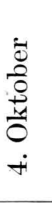 & $\begin{array}{l}\dot{0} \\
\frac{0}{0} \\
\frac{1}{4} \\
0 \\
\dot{0}\end{array}$ & $\begin{array}{l}\dot{0} \\
\frac{0}{0} \\
\frac{1}{4} \\
0 \\
0\end{array}$ & 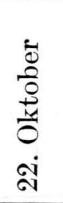 & 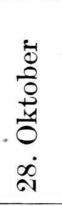 & 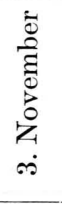 & 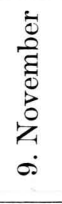 & 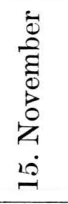 & 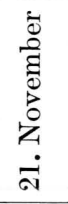 & 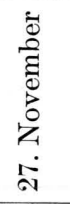 & 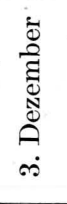 & 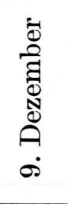 & 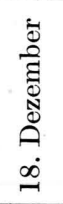 \\
\hline \multirow{2}{*}{10} & $2 n$ & 4,2 & 5,6 & 8,4 & 9,4 & 13,2 & 15,6 & 18,2 & 20,4 & 23,0 & 24,0 & 25,8 & 28,0 & 28,6 & 29,6 \\
\hline & $4 n$ & 4,0 & 5,6 & 8,2 & 9,8 & 13,4 & 16,0 & 18,6 & 20,6 & 22,2 & 24,4 & 26,8 & 28,2 & 29,4 & 30,4 \\
\hline \multirow{2}{*}{11} & $2 n$ & 4,0 & 6,4 & 8,2 & 12,4 & 14,0 & 16,2 & 18,6 & 22,4 & 25,2 & 27,2 & 29,4 & 31,5 & 32,5 & 33,0 \\
\hline & $4 n$ & 4,0 & 5,8 & 7,6 & 9,6 & 13,4 & 15,6 & 17,8 & 20,6 & 22,6 & 24,2 & 26,0 & 27,8 & 29,4 & 32,2 \\
\hline \multirow{2}{*}{15} & $2 n$ & 4,8 & 6,8 & 9,0 & 11,8 & 17,2 & 18,6 & - & - & - & - & - & - & - & - \\
\hline & $4 n$ & 4,8 & 6,0 & 9,2 & 10,2 & 17,0 & 18,8 & - & - & - & - & - & - & - & - \\
\hline \multirow{2}{*}{18} & $2 n$ & 4,8 & 6,6 & 8,8 & 12,0 & 17,6 & 17,8 & - & - & - & - & - & - & - & - \\
\hline & $4 n$ & 4,8 & 7,0 & 8,6 & 12,4 & 15,8 & 17,8 & - & - & - & - & - & - & - & - \\
\hline
\end{tabular}

Tabelle 3

Antirrhinum majus, Blattzahlzunahme bei Di- und Autotetraploiden

\begin{tabular}{|c|c|c|c|c|c|c|c|c|c|c|c|c|c|c|c|}
\hline 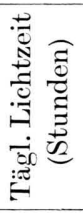 & 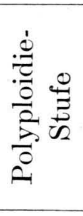 & 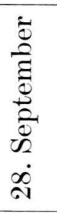 & $\begin{array}{l}\dot{0} \\
\frac{0}{0} \\
\dot{+} \\
\frac{\pi}{0} \\
\dot{+}\end{array}$ & $\begin{array}{l}\dot{0} \\
\frac{0}{0} \\
+\frac{1}{0} \\
0 \\
\dot{0}\end{array}$ & $\begin{array}{l}\frac{5}{0} \\
\frac{0}{0} \\
\frac{1}{0} \\
0 \\
0 \\
0\end{array}$ & 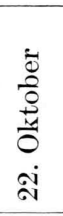 & 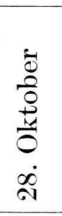 & $\begin{array}{l}\dot{0} \\
\tilde{0} \\
\tilde{\Xi} \\
\dot{0} \\
0 \\
z \\
\dot{0}\end{array}$ & 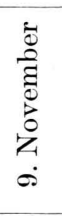 & 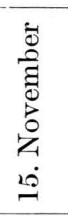 & 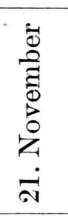 & 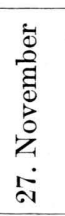 & 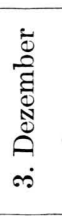 & $\begin{array}{l}\dot{0} \\
\text { है } \\
\text { हूँ } \\
\mathbb{N} \\
\stackrel{0}{0} \\
\dot{0}\end{array}$ & $\begin{array}{l}\ddot{0} \\
\stackrel{0}{0} \\
\overline{0} \\
\mathbb{N} \\
0 \\
\infty \\
\infty\end{array}$ \\
\hline \multirow{2}{*}{10} & $2 n$ & 2,0 & 4,5 & 9,0 & 12,5 & 19,0 & 23,3 & 26,0 & 29,0 & 33,5 & 36,3 & 37,3 & 39,0 & 39,0 & 40,0 \\
\hline & $4 n$ & 2,0 & 4,0 & 6,0 & 10,0 & 14,0 & 18,0 & 23,0 & 26,3 & 31,0 & 34,0 & 37,0 & 39,7 & 39,7 & 39,7 \\
\hline \multirow{2}{*}{11} & $2 n$ & 3,0 & 6,0 & 9,0 & 13,5 & 20,0 & 25,0 & 27,3 & 33,3 & 33,3 & - & - & - & - & - \\
\hline & $4 n$ & 1,8 & 4,0 & 7,3 & 10,0 & 15,3 & 19,0 & 23,0 & 28,3 & 33,0 & - & - & - & - & - \\
\hline \multirow{2}{*}{15} & $2 n$ & 4,0 & 6,0 & 10,5 & 15,0 & 16,5 & - & - & - & - & - & - & - & - & - \\
\hline & $4 n$ & 2,0 & 6,0 & 9,3 & 14,0 & 16,7 & - & - & - & - & - & - & - & - & - \\
\hline \multirow{2}{*}{18} & $2 n$ & 3,5 & 6,0 & 10,0 & 14,8 & - & - & - & - & - & - & - & - & - & - \\
\hline & $4 n$ & 2,0 & 4,5 & 7,5 & 12,7 & - & - & - & - & - & - & - & - & - & - \\
\hline
\end{tabular}


schiede erkennbar; ebenso ist die Endblattzahl bis zur 1. Blüte bei beiden dieselbe. Sowohl die Wachstumsgeschwindigkeit in der vegetativen Phase als auch die Entwicklungsgeschwindigkeit sind also durch die Polyploidie nicht beeinflußt. Jedoch scheint es, daß bei den Tetraploiden das Wachstum nach Eintritt in die reproduktive Phase relativ langsamer wird, da sonst die Verzögerung im Erscheinen von Knospen und Blüten nicht zu verstehen ist. (Da bei Hyoscyamus albus das Schossen mit der Blütẻnbildung nicht korreliert ist, ist der genave Zeitpunkt des Beginns der reproduktiven Entwicklung bei dieser Art nicht ohne weiteres festzustellen.) Bei Antirrhinum blieb die Blattzahl der Tetraploiden hinter der der Diploiden während des Wachstums zurück, das Wachstum ist also bei Polyploidie verlangsamt, wenn auch die Unterschiede gering sind und überdies gegen das Erscheinen von Knospen hin eine Tendenz zum Ausgleich besteht. Die Endblattzahl ist etwas kleiner als bei den Diploiden, die Entwicklung also relativ etwas beschleunigt. Für Xanthium dürfte dasselbe gelten wie für Antirrhinum.

\section{II.}

Die meisten der in den letzten Jahren ausgeführten Untersuchungen über die Physiologie experimentell hergestellter Polyploider gingen - bewußt oder unbewuß - von ein und derselben Fragestellung aus. In natürlichen Polyploidiereihen bestehen zwischen den Polyploiden und den nächst verwandten Diploiden oder zwischen verwandten Formen verschiedener Polyploidiestufe neben den morphologischen auch mehr oder minder große physiologische Unterschiede, wobei man in manchen Fällen gewisse Gesetzmäßigkeiten gefunden zu haben glaubt (z. B. als bekannteste eine mit dem Polyploidiegrad zunehmende Widerstandsfähigkeit gegen extremere klimatische Bedingungen). Durch Untersuchung des Verhaltens der entsprechenden physiologischen Eigenschaften bei künstlich ausgelöster Polyploidie hofft man das Zustandekommen dieser Unterschiede und dieser vermuteten Gesetzmäßigkeiten erklären zu können. Auch Unterschiede in der photoperiodischen Reaktionsweise in natürlichen Polyploidiereihen sind bereits vermutet und mit den bei experimentell hergestellten Autopoly-

10 Hereditas 30, 1 [1944].

11 Der einzige Fall eines wirklichen qualitativen Polyploidie-Effektes ist die Aufhebung der Sterilität bei vielen selbststerilen Formen nach Chromosomenverdoppelung. Hier handelt es sich aber um eine besondere Erscheinung, ploiden angeblich gefundenen Reaktionsunterschieden (Ernst) in Zusammenhang gebracht worden (A. u. D. Löve $\left.{ }^{10}\right)$.

Von diesem Blickpunkt her gesehen sind unsere Ergebnisse negativ. Die gefundenen Entwicklungsunterschiede zwischen den Di- und Autotetraploiden sind viel zu gering und dabei ohne eine wirklich eindeutige Gesetzmäßigkeit, als daß sie einen Anhalt für das Verständnis entsprechender Unterschiede bei natürlichen Di- und Autopolyploiden bieten könnten. Es fragt sich nun aber, ob die genannte Betrachtungsweise insgesamt sinnvoll ist, d. h. ob man von Versuchen über die physiologischen Eigenschaften experimentell gewonnener Polyploider überhaupt eine Erklärung der Verhältnisse bei natürlichen Polyploiden erwarten kann, und damit, ob solche Versuche einen allgemeineren Wert haben. Diese Frage ist m. E. entschieden zu verneinen, und für diese Auffassung bieten gerade einige unserer Befunde eine gute Illustration. Sie zeigen an einem relativ gut zu begrenzenden Vorgang, in wie verschiedenartiger Weise cie denselben zusammensetzenden Teilvorgänge - die ihrerseits noch hochgradig zusammengesetzter Natur sind - arf Polyploidie reagieren können, und daß sich das Verhältnis in der Reaktion dieser Teilvorgänge zudem im Laufe der Entwicklung ändern kann; sie lassen also erkennen, wie komplexer Art die Reaktion des Gesamtvorganges sein muß. Es erscheint dann von vornherein hoffnungslos, bei so komplizierten Vorgängen, wie etwa Suffproduktion, Trockensubstanzanhäufung, Längenwachstum, bei denen das Verhalten der Einzelkomponenten gänzlich unbekannt ist, allgemeinere Gesetzmäßigkeiten bezüglich der Polyploidiewirkung auffinden zu wollen. Alles bisher vorliegende Experimentalmaterial zur Morphologie wie Physiologie der Autopolyploiden läßt das auch deutlich erkennen. Die Unterschiede gegenüber den Diploiden sind durchweg von geringem Umfang und quantitativer Art ${ }^{11}$, sie können sich im Verlauf der Entwicklung verschieben und sogar umkehren, und selbst in Merkmalen oder Eigenschaften, in denen sich eine gewisse gleichbleibende Tendenz in der Richtung der Unterschiede andeutet, kommen fast immer Abweichungen von dieser Tendenz vor (siehe z.B. Györff $\mathrm{y}^{\mathbf{1 2}}$, Pirschle $\mathrm{e}^{\mathbf{1 3}}$, dort weitere Literatur).

indem im diploiden Pollen Allele zusammentreffen, die im haploiden stets getrennt sind, und ihre Wirkungen sich dabei aufheben können (vgl. D. Lewis, Nature [London] 153, 575 [1943])

12 Arb. ungar. biol. Inst. Tihany 13, 362 [1941].

13 Planta 32, 517; Z. Vererbungsl. 80, 126 u. 247 [1942]. 
Eine weitere Überlegung zeigt, daß allgemeinere Gesetzmäßigkeiten in der Merkmalsänderung nach Polyploidisierung freilich auch grundsätzlich kaum zu erwarten sind. Man muß sich klar machen, was die genetisch-entwicklungsphysiologischen Folgen der Polyploidie sind. Die blc ße Vervielfachung des Chromosomensatzes kann keine weitgehenden direkten Folgen für die Merkmalsausprägung haben; Änderungen der Merkmalsausprägung bei Polyploiden werden allermeist auf indirekten Wirkungen der Chromosomenvermehrung beruhen. Solcher Wirkungen gibt es zweierlei: 1. Durch die Vermehrung der Chromosomen wird der Kern- und Zellteilungsmechanismus über das normale Maß belastet. 2. Verschiedene Gene vermehren ihre Wirkung mit der Vermehrung ihrer Zahl verschieden stark, wodurch das Gleichgewicht der Genwirkungen bei Polyploiden verändert wirc 14. Der Umfang der entwicklungsphysiologischen Folgen der ersten Wirkung dürfte beschränkt sein; sie kann, da die Kern- und Zellteilung der Hauptregulator des Wachstums ist, zu einer Verlangsamung desselben und ferner zum Auftreten chromosomaler Abweichungen führen, und wahrscheinlich ist die sehr häufig ang€g€bene, auch in unseren Versuchen beobachtete Wachstumsverlangsamung von experimentell hergestellten Polyploiden sowie das ebenfalls häufige, fast regelmäßige, Vorkommen aberranter Formen in ihrer Nachkommenschaft wenigstens teilweise mit dieser Polyploidiewirkung zu erklären. Alle anderen Veränderungen der morphologischen wie physiologischen Eigenschaften nach Polyploidisierung können auf die als zweite genannte Wirkung, und nur auf diese, zurückg eführt werden ${ }^{15}$. Die Polyploidie ist in erster Linie also eine Frage der

14 Vgl. zu dieser Frage die theoretische Studie von G. Melchers, diese Z.1,160 [1946]. — Die soeben erwähnte Polyploidiewirkung bei selbststerilen Pflanzen gehört als Sonderfall zur zweiten Gruppe der Polyploidiewirkungen; für die Genphysiologie ist sie von besonders großem Interesse.

15 Ernst (a. a. O.) hat zur Erklärung für das Zustande-
Genwirkung, und wirkliche Fortschritte in der Erforschung von Polyploidiewirkungen sind nur zu erwarten, wenn man die Arbeit von dieser Grundlage her betreibt.

Es ist bemerkenswert, daß für diese, auf Grund prinzipieller Überlegungen hin zu fordernde Auffassung der Polyploidiewirkung seit Jahren ein ausgezeichnetes Experimentalmaterial vorliegt. Law rence $u$. Scott-Moncrieff ${ }^{\mathbf{1 6}}$ haben bei Dahlien verschiedener Autopolyploidiestufe gezeigt, daß gewisse Gene das Maximum ihrer Wirkung bereits bei der simplexen Stufe erreichen, die Wirkung anderer sich dagegen bis zur duplexen, triplexen, quadruplexen Stufe steigern läßt. Auf polyploider Basis lassen sich daher Stärkegrade von Genwirkungen kombinieren, deren Kombination auf diploider nicht möglich ist. Diese Befunde sind an sog. , , alten“ Polyploiden gewonnen; es wäre erwünscht — wenn auch am Ausgang solcher Versuche kein Zweifel besteht - , sie an frisch hergestellten zu wiederholen. Jedenfalls liegt in dieser Richtung und nicht in der Untersuchung von Polyploiden unbekannten Genotyps die künftige Hauptaufgabe dieses Zweiges der experimentellen Polyploidieforschung. Auch die Frage nach dem Zustandekommen der Unterschiede zwischen natürlichen Poly- und Diploiden beantwortet sich auf Grund der hier entwickelten Überlegungen grundsätzlich von selbst. Diese Unterschiede sind keine ,,direkte“ Folge der Polyploidie. Sie müssen durch Selektion auf der polyploiden Basis entstanden sein; da Polyploidie eine Erweiterung des Selektionsmaterials mit sich bringt, ist das Vorhandensein von Merkmalen und Leistungen bei natürlichen Polyploiden, die bei den nächstverwandten Diploiden nicht zu finden sind, grundsätzlich ebenfalls leicht zu verstehen.

kommen der Wachstumshemmungen bei autotetraploidem Antirrhinum Mitwirkung des Plasmas angenommen. Auch wenn es sich bei diesen Hemmungen um einen qualitativen Polyploidieeffekt handelte, läge für solche Annahme keinerlei Veranlassung vor.

${ }_{16}$ J. Genetics 30, 155 [1935]. 